\title{
Descrição de sintomas ginecológicos e obstétricos em mulheres ribeirinhas da Amazônia Brasileira
}

\author{
Mariane Benedicto de Souza1 https://orcid.org/0000-0003-3139-4084 \\ José Anibale Rodrigues Junior ${ }^{1}$ https://orcid.org/0000-0002-5618-8028 \\ Jhenifer Nataly Moura França' ${ }^{1}$ https://orcid.org/0000-0002-3334-5422 \\ Caio Hartman ${ }^{1}$ https://orcid.org/0000-0001-5278-1599 \\ Fabiana Moreira Passos Succi ${ }^{1}$ https://orcid.org/0000-0001-5337-1292 \\ Kátia Piton Serra1 $\quad$ https://orcid.org/0000-0003-4117-670X
}

\section{$\underline{\text { RESUMO }}$}

Objetivo: Descrever os principais sintomas e diagnósticos dos atendimentos ginecolóticos e obstétricos realizados às mulheres ribeirinhas em Santarém-PA durante a Expedição Barco da Saúde 2018. Materiais e Métodos: Estudo descritivo composto por amostra de 134 mulheres residentes em comunidades ribeirinhas do município de Santarém-PA, que foram atendidas durante expedição realizada de 22 a 30 de julho de 2018. Resultados: Foram realizados 134 atendimentos em ginecologia-obstetrícia. A média de idade foi de 30,9 anos. Os principais diagnósticos encontrados foram: pré-natal 33 (24,6\%), dor pélvica 28 (20,9\%), vulvovaginite 15 $(11,2 \%)$, menstruação irregular $14(10.4 \%)$, dismenorreia $9(6,7 \%)$, sintomas mamários $9(6,7 \%)$, infecção sexualmente transmitida $6(4,5 \%)$, infecção do trato urinário $6(4,5 \%)$, planejamento familiar $5(3,7 \%)$ e outros $9(6,7 \%)$. Entre as adolescentes predominaram os sintomas menstruais com $7(46,7 \%)$ casos; entre as adultas predominaram pré-natal com 31 (26\%) casos e dor pélvica com 28 (23,5\%). Conclusão: É importante conhecer as necessidades dessas comunidades para subsidiar políticas de saúde voltadas para a atenção humanizada à mulher ribeirinha.

Palavras chave: avaliação de sintomas, saúde da mulher, grupos populacionais, direito à saúde.

\section{INTRODUÇ̃̃O}

O Sistema Único de Saúde (SUS) [1] foi implantado há três décadas assegurando o direito pleno e universal ao acesso à saúde em todo território brasileiro nos diversos níveis de complexidade [2]. Porém, em algumas regiões o do Brasil, o acesso aos serviços é uma grande barreira, o que dificulta o ingresso de muitos usuários e configura um desafio para a saúde pública [3].

O Brasil possui uma grande extensão territorial e possui povos com diferentes designações e culturas [4]. A área urbana proporciona fácil acesso a transportes, tecnologia, educação, saúde e tratamentos. Por outro lado, a população que vive às margens dos rios, denominadas ribeirinhas, apresenta grandes dificuldades na obtenção e uso destes serviços. Tal circunstância restringe o acesso a transportes, alimentos e tecnologias, além de acesso à educação, atendimentos médicos e fármacos [5]. Esta população tem dificuldade para atendimento de saúde decorrente do baixo poder econômico e alcance limitado às unidades de saúde, as quais estão dispostas apenas nas regiões centrais dos estados, e ainda há apenas uma forma de transporte, a fluvial, com restrições de tempo e disponibilidade [6]. Em relação à saúde da mulher, observa-se

Faculdade São Leopoldo Mandic, Curso de Medicina. Rua José Rocha Junqueira, 13, 13041-755, Campinas, SP, Brasil. Correspondência para: SERRA KP. E-mail: <katiapserra@gmail.com> 
uma situação ainda pior. As mulheres inseridas neste contexto social carecem de informações básicas sobre educação em saúde e principalmente sobre direito reprodutivo e planejamento familiar. O papel da mulher na sociedade ribeirinha está relacionado a subordinação perante o seu parceiro [7].

Diante deste cenário, ações com o objetivo de promover saúde e prevenir doenças se tornam prioridade. Porém, existem poucos estudos que demonstrem os principais problemas relacionados especificamente à saúde reprodutiva da mulher ribeirinha.

O objetivo do presente trabalho foi descrever os principais sintomas e diagnósticos dos atendimentos ginecológicos e obstétricos às mulheres ribeirinhas da região do Rio Tapajós - Santarém - PA durante a expedição "Barco da Saúde São Leopoldo Mandic", 2018.

\section{MÉTODOS}

Estudo descritivo composto por uma amostra de 134 mulheres residentes em comunidades ribeirinhas do município de Santarém - PA, que foram atendidas durante expedição voluntária de saúde realizada de 22 a 30 de julho de 2018.

Foi submetido e aprovado pelo Comitê de Ética em pesquisa sob o número 3.987.173, CAAE 30352220.7.0000.5374.

\section{Coleta e análise dos dados}

A coleta de informações na área de ginecologia e obstetrícia foi realizada de maneira padronizada através de uma ficha de atendimento destinada para este fim. A ficha foi elaborada pela equipe em reuniões prévias à expedição, pelos próprios alunos e professores.

Os dados dos atendimentos foram extraídos das fichas clínicas e posteriormente tabulados no Microsoft Excel. As variáveis numéricas foram expressas em média. As variáveis categóricas foram expressas em porcentagem.

\section{RESULTADOS}

Foram realizados 134 atendimentos em ginecologia e obstetrícia. A média de idade das mulheres foi de 30,9 anos, com idade mínima de 4 e máxima de 56 anos.
Os principais diagnósticos nos atendimentos de ginecologia-obstetrícia foram: Pré-natal $33(24,6 \%)$ casos, dor pélvica 28 (20,9\%), vulvovaginite 15 (11,2\%), menstruação irregular $14(10,5 \%)$, dismenorreia 9 $(6,7 \%)$, sintomas mamários (mastalgia, nódulo e mama acessória) $9(6,7 \%)$, infecção sexualmente transmitida (IST) 6 (4,5\%), infecção do trato urinário (ITU) 6 (4,5\%), planejamento familiar $5(3,7 \%)$ e outros diagnósticos 9 $(6,7 \%)$ casos, como demonstrado no gráfico 1.

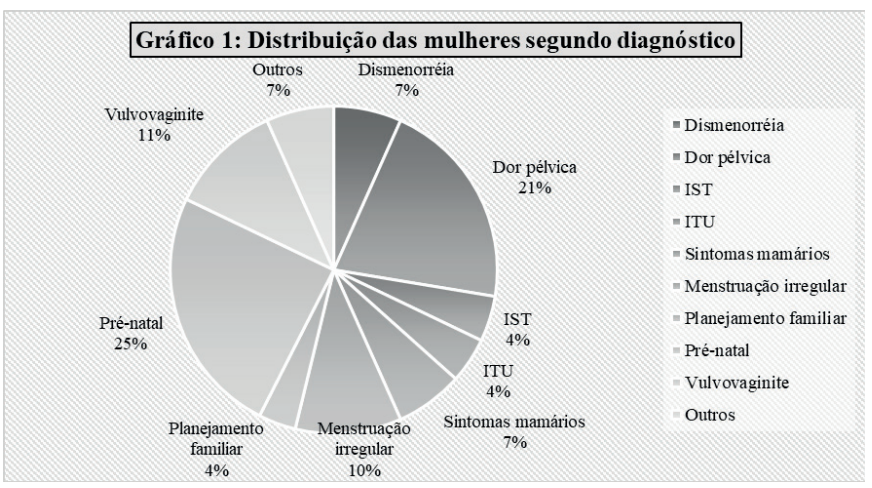

As gestantes apresentaram média de idade de 24,6 anos, sendo 14 anos a mais jovem e 42 anos a mais velha.

Apenas 15 pacientes apresentaram menos de 18 anos de idade ao atendimento. Entre esse grupo houve uma prevalência de queixas menstruais, que juntas somaram $46,7 \%$ da procura pelo atendimento. A distribuição dos sintomas nesse grupo foi: 4 casos $(26,7 \%)$ dismenorreia, 3 (20\%) menstruação irregular, 3 (20\%) ITU, 2 (13,3\%) vulvovaginite, 1 (6,7\%) planejamento familiar e $2(13,3 \%)$ pré-natal (idades de 14 e 17 anos) (tabela 1).

Entre as mulheres com 18 anos ou mais predominaram a procura por pré-natal 31 (26\%) casos e dor pélvica 28 (23,5\%). Nesse grupo apareceram os sintomas mamários (mastalgia, nódulo e mama acessória) com 9 (7,6\%) casos (tabela 1). 
Tabela 1 - Distribuição dos principais diagnósticos encontrados por idade

\begin{tabular}{|l|c|c|c|c|}
\hline \multirow{2}{*}{ Diagnóstico } & \multicolumn{2}{|c|}{$<18$ anos } & \multicolumn{2}{c|}{$>18$ anos } \\
\hline \hline & $N$ & $\%$ & $N$ & $\%$ \\
\hline Dismenorreia & 4 & 26,7 & 5 & 4,2 \\
\hline Dor pélvica & - & - & 28 & 23,5 \\
\hline *IST & - & - & 6 & 5 \\
\hline **ITU & 3 & 20 & 3 & 2,5 \\
\hline $\begin{array}{l}\text { Sintomas } \\
\text { mamários }\end{array}$ & - & - & 9 & 7,6 \\
\hline $\begin{array}{l}\text { Menstruação } \\
\text { irregular }\end{array}$ & 3 & 20 & 11 & 9,3 \\
\hline $\begin{array}{l}\text { Planejamento } \\
\text { familiar }\end{array}$ & 1 & 6,7 & 4 & 3,4 \\
\hline Pré-natal & 2 & 13,3 & 31 & 26 \\
\hline Vulvovaginite & 2 & 13,3 & 13 & 10,9 \\
\hline Outros & - & - & 9 & 7,6 \\
\hline TOTAL & 15 & 100 & 119 & 100 \\
\hline
\end{tabular}

Nota: *IST: infecção sexualmente transmitida; **ITU: infecção do trato urinário. Sinal convencional utilizado: - Dado numérico igual a zero não resultante de arredondamento

\section{DISCUSSÃO}

Entre o universo de mulheres atendidas, houve uma predominância de mulheres adultas, apenas 15 $(11,2 \%)$ tinham menos de 18 anos.

Apesar das orientações sobre planejamento familiar nas comunidades, foi baixa a procura para anticoncepção (apenas 3,7\% dos atendimentos). Entre as 5 mulheres atendidas com esse objetivo, apenas 1 era adolescente. Em pesquisa qualitativa realizada em 2016, dos Santos et al. [7] descrevem mulheres conscientes de sua saúde sexual e reprodutiva, apesar da baixa escolaridade, e uso amplo de condom. O presente estudo não foi desenhado para essa finalidade, apenas descreve o observado durante os atendimentos, porém, chamou a atenção dos pesquisadores a baixa procura por métodos contraceptivos.

As queixas entre as adolescentes foram predominantemente sobre questões menstruais, típicas da faixa etária. Houve apenas 2 gestantes nesse grupo, o que surpreendeu à equipe, que esperava encontrar mais grávidas adolescentes. Segundo os poucos relatos da literatura, a maioria das mulheres ribeirinhas se casa e engravida na adolescência e é considerado uma necessidade biológica, e lhe são atribuídos papéis perante a sociedade, entre eles garantir a organização do lar $[8,9]$.

Entre as mulheres adultas a procura mais frequente foi para realização de pré-natal e por dor pélvica. Esse sintoma chamou atenção pela sua frequência (28 mulheres, 23,5\% da população a partir de 18 anos). Aparentemente esse sintoma não estaria relacionado a IST nem ITU, pois a prevalência desses diagnósticos foi de $5 \%$ (6 casos) e 2,5\% (3 casos) respectivamente. Também não foi realizada investigação para parasitoses, o tratamento foi empírico nos casos clinicamente suspeitos. De fato, para elucidar essa causa é necessário outro estudo com desenho apropriado. Uma característica desse grupo foi o surgimento de sintomas mamários, que não apareceram entre as adolescentes.

Este foi um estudo descritivo sobre os principais sintomas e diagnósticos realizados ao atendimento às mulheres ribeirinhas em uma ação voluntária. Não foi desenhado para fazer diagnóstico dos principais problemas em saúde da mulher nesta população. Porém a divulgação desses problemas levantados é importante para que estudos sejam pensados no sentido de identificar as principais carências dessas mulheres. Espera-se que o levantamento dos dados epidemiológicos e vivências em saúde compartilhadas com essa população possam contribuir para uma melhor percepção da mulher ribeirinha, suas demandas e sobre a prática de cuidado voltada aos povos tradicionais. Assim será possível desenhar políticas de saúde voltadas às necessidades específicas dessa população.

\section{CONCLUSÃO}

Estudos com desenho específico para identificação dos principais problemas inerentes á saúde da mulher ribeirinha são necessários para que sejam desenvolvidas políticas de saúde que atendam às necessidades dessa população tradicicional. 


\section{REFERÊNCIAS}

1. Brasil. Constituição (1988). Constituição da República Federativa do Brasil. Brasília, DF: Senado Federal: Centro Gráfico, 1988. Seção II - Da Saúde. Disponível em: <http://conselho.saude. gov.br/web_sus20anos/20anossus/legislacao/constituicaofederal. pdf>. Acesso em 16 Jul. 2020.

2. Peiter CC, Santos JLG, Lanzoni GMM, Mello ALSF, da Costa MFBNA, Andrade SR. Redes de atenção à saúde: tendências da produção de conhecimento no Brasil. Esc Anna Nery. 2019 jan;23(1): e20180214. http://dx.doi.org/10.1590/2177-9465-EAN-2018-0214

2. Puccini PT. As unidades de assistência médica ambulatorial (AMA) do Município de São Paulo, Brasil: condições de funcionamento e repercussões sobre a atenção básica no Sistema Único de Saúde 2006. Cad Saúde Pública. 2008 dez;24(12):2755-66.

3. Morim J. Povos e Comunidades Tradicionais. Disponível em:https://pesquisaescolar.fundaj.gov.br/pt-br/artigo/povos-ecomunidades-tradicionais/. Acesso em: 16 Jul. 2020A.

4. Morim J. Ribeirinhos. Disponível em: https:// pesquisaescolar.fundaj.gov.br/pt-br/artigo/ribeirinhos/. Acesso em: 16 Jul. 2020B.

5. Gama ASM, Fernandes TG, Parente RCP, Secoli SR. Inquérito de saúde em comunidades ribeirinhas do Amazonas, Brasil. Cad. Saúde Pública. 2018;34(2):e00002817.

6. dos Santos JKL, Santos SC, Sales APA, Araújo OMR, Batiston AP. Percepção da mulher ribeirinha sobre os cuidados com a saúde sexual e reprodutiva. Investigação Qualitativa em Saúde. 2016 jul;2:710-17.

7. Silva MGSN, Ramos TCA. Práticas alternativas para a saúde da mulher ribeirinha. Revista de Educação, Cultura e Meio Ambiente- Edição Especial Mulher. 1998 dez;14(II):1-11.

8. da Silva LR, da Silva RF. Conhecimento, atitudes e crenças de mulheres ribeirinhas frente à concepção e contracepção. Rev enferm UFPE. 2009 out/dez;3(4):972-80. 